IMMUNOTHERAPY

\section{Down the drain?}

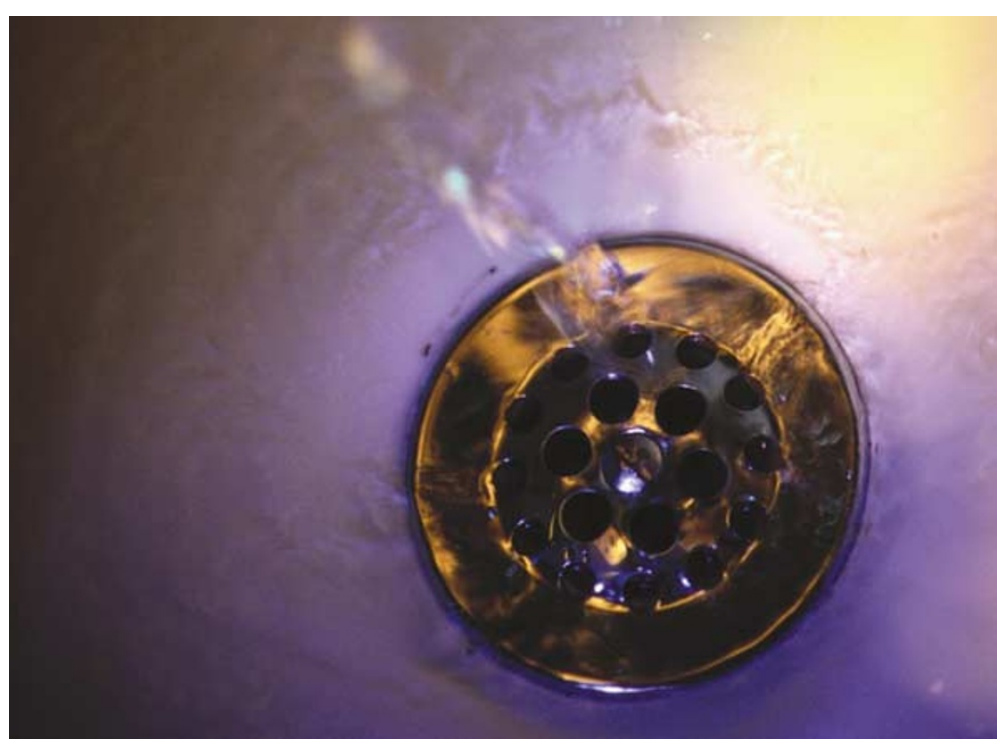

The ability of interleukin-2 (IL-2) to induce non-specific killing of tumour cells has led to its use as a treatment for both malignant melanoma and renal-cell carcinoma. However, hopes for the therapy were being washed away by the systemic toxicity of high-dose IL-2; $65 \%$ of patients develop capillaryleak syndrome (increased vascular permeability) and have to stop therapy. What component of IL-2 is responsible for unblocking the plugholes between vascular endothelial cells and allowing the draining of intravascular fluids to extravascular spaces?

Epstein et al. produced a series of overlapping or contiguous peptides of human IL-2, which were conjugated with the monoclonal antibody Lym1 (which recognizes HLADR10 on the surface of human lymphoma cells). Nude mice bearing xenografts of Raji lymphoma cells received intravenous injections of the various antibody conjugates, then 2 hours later were injected with radiolabelled Lym 1 as a tracer. The biodistribution of the tracer antibody was analysed 72 hours later, as an indicator of effects on vasopermeability. An antibody conjugate containing amino acids $22-58$ of IL-2 was shown to enhance tumour uptake of the tracer to the same extent as antibody-conjugated fulllength IL-2. This peptide was therefore named permeability-enhancing peptide (PEP). The PEP region of IL-2 partially overlaps with the receptor-binding site of the molecule, so the PEP region was tested for cytokine activity, but was unable to induce the proliferation of IL-2dependent HT-2 cells.

So, the authors have separated the cytokine activities of IL-2 from its effects on the vasculature. If this information can be converted into a genetically modified form of IL-2 that retains cytokine function but

\title{
New directions for tumour therapy
}

Dendritic cell (DC)-based antitumour vaccines have been developed that depend on the ability of DCs to present tumour antigens to $\mathrm{T}$ cells and enhance T-cellmediated antitumour responses. Vaccines that are being tested at present in clinical trials use DCs that are loaded with tumour antigens ex vivo before being re-infused into the recipient. Despite the success of this approach in generating antitumour immune responses, the long-term results have been less promising. Whartenby and colleagues now describe a new bonemarrow transplantation (BMT)-based strategy for treating established tumours, in which haematopoietic stem cells (HSCs) are transduced with genes that encode tumour antigens: these then differentiate in vivo.

HSCs were first transduced with lentiviral vectors expressing green-fluorescent protein (GFP) and were then transplanted into lethally irradiated mice. A large proportion of DCs that developed in the lymphoid organs of these recipients expressed GFP, indicating that transduced HSCs develop into DCs that express the GFP transgene and successfully traffic to the peripheral lymphoid organs.

To see if the transduced DCs could activate antigen-specific responses, the authors carried out BMTs with HSCs that had been transduced with control or tumour-antigen (haemagglutinin; HA)encoding lentiviruses in irradiated mice, which also received transgenic $\mathrm{T}$ cells with HA-specific T-cell receptors. Ten days before BMT, the recipients were injected with HA-expressing lymphoma (A20-HA) cells. In addition, 3 weeks after BMT, Flt3 ligand and antibodies specific for $\mathrm{CD} 40$ were administered (to activate DCs), as well as mature $\mathrm{T}$ cells from HA-transgenic mice (to help overcome the induction of central tolerance, which could occur following repopulation of the thymus with antigen-expressing DCs).

What was the outcome of this approach, and did it result in therapeutic benefit for established tumours? HA-specific T cells were activated and proliferated in recipients of HA-transduced HSCs, when given in combination with Flt3 ligand, CD40-specific antibodies and mature
T cells. The expanded T-cell population had effector function and could be expanded in mice even 1 year after transplantation. This strategy was shown to be more successful than administration of $e x$-vivo-generated transduced DCs in mice with established tumours. The treatment resulted in the long-term survival of $\sim 50 \%$ of treated mice, even when administered $\mathrm{T}$ cells were from tumour bearing, non-transgenic mice.

As the authors conclude, although this method would need to be adapted for use in the clinic - and even then it could only be applied to tumours with known antigens — this work highlights a potential new approach for inducing antigen-specific tumour immunity using antigen-genetransduced HSCs.

$$
\begin{array}{r}
\text { Jenny Buckland } \\
\text { Senior Editor, } \\
\text { Nature Reviews Immunology }
\end{array}
$$

(2) References and links ORIGINAL RESEARCH PAPER Cui, Y. et al. Immunotherapy of established tumors using bone marrow transplantation with antigen gene-modified hematopoietic stem cells. Nature Med. 9, 952-958 (2003) 